\title{
WUSCHEL-related Homeobox genes in Populus tomentosa: diversified expression patterns and a functional similarity in adventitious root formation
}

Bobin Liu' ${ }^{1,2}$, Lin Wang ${ }^{2,3}$, Jin Zhang ${ }^{2}$, Jianbo Li ${ }^{2}$, Huanquan Zheng ${ }^{4}$, Jun Chen ${ }^{2,3^{*}}$ and Mengzhu Lu ${ }^{1,2,3^{*}}$

\begin{abstract}
Background: WUSCHEL (WUS)-related homeobox (WOX) protein family members play important roles in the maintenance and proliferation of the stem cell niche in the shoot apical meristem (SAM), root apical meristem (RAM), and cambium (CAM). Although the roles of some WOXs in meristematic cell regulation have been well studied in annual plants such as Arabidopsis and rice, the expression and function of WOX members in woody plant poplars has not been systematically investigated. Here, we present the identification and comprehensive analysis of the expression and function of WOXs in Populus tomentosa.

Results: A genome-wide survey identified 18 WOX encoding sequences in the sequenced genome of Populus trichocarpa (PtrWOXs). Phylogenetic and gene structure analysis revealed that these 18 PtrWOXs fall into modern/WUS, intermediate, and ancient clades, but that the WOX genes in $P$. trichocarpa may have expanded differently from the WOX genes in Arabidopsis. In the P. trichocarpa genome, no WOX members could be closely classified as AtWOX3, AtWOX6, AtWOX7, AtWOX10, and AtWOX14, but there were two copies of WOX genes that could be classified as PtrWUS, PtrWOX2, PtrWOX4, PtrWOX5, PtrWOX8/9, and PtrWOX11/12, and three copies of WOX genes that could be classified as PtrWOX 1 and PtrWOX 13. The use of primers specific for each PtrWOX gene allowed the identification and cloning of 18 WOX genes from $P$. tomentosa (PtoWOXs), a poplar species physiologically close to $P$. trichocarpa. It was found that PtoWOXs and PtrWOXs shared very high amino acid sequence identity, and that PtoWOXs could be classified identically to PtrWOXs. We revealed that the expression patterns of some PtoWOXs were different to their Arabidopsis counterparts. When PtoWOX5a and PtoWOX11/12a, as well as PtoWUSa and PtoWOX4a were ectopically expressed in transgenic hybrid poplars, the regeneration of adventitious root (AR) was promoted, indicating a functional similarity of these four WOXs in AR regeneration.
\end{abstract}

Conclusions: This is the first attempt towards a systematical analysis of the function of WOXs in P. tomentosa. A diversified expression, yet functional similarity of PtoWOXs in AR regeneration is revealed. Our findings provide useful information for further elucidation of the functions and mechanisms of WOXs in the development of poplars.

Keywords: Adventitious root, Expression, Homeobox, Populus, WOX, Wuschel-related

\footnotetext{
* Correspondence: chenjun@caf.ac.cn; lumz@caf.ac.cn

${ }^{2}$ State Key Laboratory of Tree Genetics and Breeding, Research Institute of

Forestry, Chinese Academy of Forestry, Beijing 100 091, China

'Key Laboratory of Forest Genetics \& Biotechnology of Ministry of Education,

Nanjing Forestry University, Nanjing 210 037, China

Full list of author information is available at the end of the article
} 


\section{Background}

Homeobox (HB) proteins were first discovered in Drosophila. They are a superfamily of transcriptional factor proteins containing a conserved 60-amino acid homeodomain (HD). HB proteins have been found in all eukaryotic organisms tested [1-3]. In plants, a great number of HD-containing transcriptional factors have been identified in both monocots and dicots [4], with KNOTTED1 being the first identified HD-containing protein [5]. The HB protein superfamily is classified into six families based on HD sequence, location, association with other functional domains, and the protein size and structure. These classifications are: homeodomain-leucine (HD-Zip), plant homeodomain (PHD)-finger, BELL, zinc finger-homeodomain (ZF-HD), WUSCHEL (WUS)related homeobox (WOX), and KNOTTED1-like-homeobox (KNOX) [4].

The WOX family of HB proteins is distinguished by the phylogenetic relatedness of its homeodomain, and is a plant specific HB transcription factor family [6]. The Arabidopsis genome encodes at least 15 WOX proteins, and these are classified into three clades: [7] a modern/WUS clade including WUS and AtWOX1-7; an intermediate clade including AtWOX8, 9, 11, and 12; and an ancient clade containing AtWOX10, 13, and 14 [6,8]. Arabidopsis WOXs regulate key developmental processes including stem cell maintenance in the SAM, RAM, and CAM, embryo apical-basal polarity patterning, and lateral organ development [8]. AtWUS, a member of the modern WOX clade, is expressed specifically in the organizing center of the SAM, and is required for maintaining stem cells via a feedback loop with CLV3, a peptide ligand that interacts with leucine-rich repeat receptor kinases in neighboring cells to restrict the size of the SAM in Arabidopsis [9]. AtWOX5, also a member of the modern WOX clade, is expressed in root quiescent centre $(\mathrm{QC})$ cells surrounded by the stem cells [10]. The AtWOX5 protein is essential for stem cell maintenance via a negative feedback signal provided by CLE40 [11]. AtWWOX4 is strongly expressed in the CAM, and regulates vascular stem cell maintenance $[12,13]$. AtWOX2 is expressed in zygotes, and is involved in regulating cell fate in the apical and basal lineage of developing embryos [14]. AtWOX3/PRS1 is involved in lateral organ development through recruiting organ founder cells forming the lateral domain in Arabidopsis [15]. AtWOX6 is expressed abundantly in developing ovules, and is involved in either ovule patterning or differentiation [16]. With regards to stem cell maintenance in the SAM and RAM, it is noteworthy that AtWUS and AtWOX5 are interchangeable [10]. Furthermore, the function of AtWOX3 in lateral organ development can be fully complemented by AtWUS [15], and partially complemented by AtWOX4 [17]. Taken together, it appears there is a common mechanism of action among modern WOX proteins. It has recently been demonstrated that members of the modern WOX clade have evolved, through the acquisition of a conserved WUS-box, a repressive activity important for leaf blade outgrowth regulation [8].

For the intermediate WOXs, AtWOX8, co-expressed with AtWOX2 in the zygote, is required for normal development of the pre-embryo [14]. Similar to AtWOX8 in Arabidopsis, $\mathrm{PaWOX8/9}$ is highly expressed at the early zygotic growth stages and the later embryo stages in Picea abies [18]. The AtWOX9 gene is involved in maintaining cell division and preventing premature differentiation in the Arabidopsis SAM [19]. The expression patterns and function of AtWOX11 and 12 are currently unknown in Arabidopsis, but $O s W O X 11$ in rice is reported to be required for activating shoot-borne root development by directly repressing $R R 2$, a cytokinin type-A responsive regulator gene [20].

With regards to the most conserved ancient plant WOX proteins, the expression and function of AtWOX10 is unknown. Although AtWOX13 is expressed ubiquitously it is most strongly expressed in developed flowers and young siliques, where it is involved in the promotion of replum formation during fruit development [21]. AtWOX14 is thought to prevent premature differentiation of primary roots, lateral roots, and floral organs [22]. AtWOX14 is predominantly expressed in vascular tissues and acts redundantly with AtWOX4 in vascular cell differentiation [23].

Poplars are a widely distributed group of economic plants. As a rapid growth species, vegetative growth leads to higher production of plant biomass [24], thus poplars are considered as feedstocks for bioenergy and timber $[25,26]$. Populus tomentosa is a Chinese native poplar species with a high economic value and is widely planted in northern China. Because of the key roles that WOX proteins play in stem cell maintenance and lateral organ development, WOX proteins are potential targets for better and faster growth of $P$. tomentosa. Here, we identify 18 WOX encoding genes in P. tomentosa (PtoWOXs). We provide a comprehensive analysis of the expression and function of the PtoWOXs. We reveal that although members of all three WOX clades exist in P. tomentosa, PtoWOXs expanded differently from those of Arabidopsis. The expression of PtoWOXs was found to differ from their Arabidopsis counterparts, with many showing significant expression in the roots, and being inducible in the regeneration of adventitious roots (ARs). Furthermore, we report that the ectopic expression of PtoWOXs from both modern and intermediate WOX clades promotes the regeneration of ARs.

\section{Results}

Identification and phylogenetic analysis of WOX genes in Populus trichocarpa and $P$. tomentosa

The $P$. tomentosa genome has not been sequenced. Therefore, to initiate functional analysis of WOXs in 
P. tomentosa we searched for WOX genes in the sequenced genome of $P$. trichocarpa, a poplar species physiologically close to $P$. tomentosa. We used all 15 known Arabidopsis WOX protein sequences as queries to blast against the $P$. trichocarpa genome database. Eighteen WOX gene sequences were identified in the $P$. trichocarpa genome; this is similar to the number of WOXs found in Arabidopsis (15) [7], Oryza sativa (13) [27], Zea mays (18) [28], and Vitis vinifera (12) [28]. The putative WOX proteins in $P$. trichocarpa (PtrWOXs) ranged from 171 to 390 amino acids in length (Table 1). A phylogenetic tree of WOX proteins from P. trichocarpa and Arabidopsis was generated based on the full WOX amino acid sequences (Figure 1, left side). Similar to the HD sequence based phylogeny of known plant WOX proteins [29], the 18 PtrWOXs fell into three major branches: the modern/WUS clade, the intermediate clade, and the ancient clade; these were similar to their Arabidopsis counterparts (Figure 1, left). The modern/WUS clade contained 11 PtrWOXs; these could be divided into five subclasses based on their relationship with their Arabidopsis counterparts. These were named as PtrWUS, PtrWOX1, PtrWOX2, PtrWOX4, and PtrWOX5. The intermediate clade consisted of four PtrWOXs; these were classified into two subclasses: PtrWOX8/9, and PtrWOX11/12. The ancient clade contained only one subclass: PtrWOX13. This classification was further confirmed by the exon/intron organization of the WOX coding sequences in $P$. trichocarpa and Arabidposis (Figure 1, right). With the exception of PtrWOX8/9a and PtrWOX11/12b, all other PtrWOX genes shared a similar intron/exon organization in the size and arrangement of introns and exons with their corresponding AtWOX counterparts.

All PtrWOXs contained highly conserved HD residues (Additional file 1: Figure S1A). A conserved WUS-box domain (TLXLFP) located downstream of the HD domain [7] was also found in members of the modern clade, but not in the other clades (Additional file 1: Figure S1B). In addition, an EAR-like domain was present in the C-terminal ends of PtrWUSa, PtrWUSb, PtrWOX5a, and PtrWOX5b (Additional file 1: Figure S1C).

Although the 18 PtrWOXs could be classified into modern/WUS clade, intermediate, or ancient clades, it is interesting that PtrWOXs may have expanded differently in Arabidopsis (Figure 1 left). Two copies of PtrWOXs could be classified as WUS (PtrWUSa and b), WOX2 (PtrWOX2a and b), WOX4 (PtrWOX4a and b), WOX5 (PtrWOX5a and b), WOX8/9 (PtrWOX8/9a and b), and WOX11/12 (PtrWOX11/12a and b), and three copies of PtrWOXs could be classified as WOX1 (PtrWOX1a, b, and c) and WOX13 (PtrWOX13a, b, and c). However, no PtrWOXs were closely related to AtWOX3, AtWOX6, AtWOX7, AtWOX10, and AtWOX14 in the phylogenetic tree (Figure 1, left). Similarly, close orthologs of AtWOX7,

Table 1 Summary of the PtrWOX gene family

\begin{tabular}{|c|c|c|c|c|c|}
\hline Gene name & Locus & Genomic position & Arabidopsis WOXs* & Length & Size $(k D)$ \\
\hline PtrWUSa & Potri.005G114700 & Chr05: 8858336-8859980 & AT2G17950 & 264 & 30.004 \\
\hline PtrWUSb & Potri.007G012100 & Chr07: 958955-960287 & AT2G17950 & 264 & 29.274 \\
\hline PtrWOX1a & Potri.012G047700 & Chr12: 4446344-4449088 & AT3G18010 & 387 & 43.851 \\
\hline PtrWOX1b & Potri.015G039100 & Chr15: 3566414-3569217 & AT3G18010 & 374 & 42.326 \\
\hline PtrWOX1c & Potri.010G111400 & Chr10: $13009243-13010950$ & AT3G18010 & 316 & 35.699 \\
\hline PtrWOX2a & Potri.001G237900 & Chr01: $24918823-24920230$ & AT5G59340 & 245 & 27.877 \\
\hline PtrWOX2b & Potri.009G029200 & Chr09: 3976470-3977823 & AT5G59340 & 246 & 27.601 \\
\hline PtrWOX4a & Potri.002G124100 & Chr02: 9309765-9311320 & AT1G46480 & 213 & 24.417 \\
\hline PtrWOX4b & Potri.014G025300 & Chr14: 2169818-2171379 & AT1G46480 & 213 & 24.463 \\
\hline PtrWOX5a & Potri.008G065400 & Chr08: 3963469-3964104 & AT3G11260 & 181 & 21.009 \\
\hline PtrWOX5b & Potri.010G192100 & Chr10: $18688337-18689050$ & AT3G11260 & 171 & 19.615 \\
\hline PtrWOX8/9a & Potri.004G051600 & Chr04: 4008260-4010297 & AT2G33880 & 390 & 43.072 \\
\hline PtrWOX8/9b & Potri.011G061400 & Chr11: 5466036-5468417 & AT2G33880 & 377 & 41.601 \\
\hline \multirow[t]{2}{*}{ PtrWOX11/12a } & Potri.013G066900 & Chr13: 5239050-5240692 & AT3G03660 & 255 & 28.086 \\
\hline & & & AT5G17810 & & \\
\hline \multirow[t]{2}{*}{ PtrWOX11/12b } & Potri.019G040800 & Chr19: 4693429-4695238 & AT3G03660 & 226 & 25.001 \\
\hline & & & At5G17810 & & \\
\hline PtrWOX13a & Potri.005G101800 & Chr05: 7788135-7790958 & AT4G35550 & 248 & 27.984 \\
\hline PtrWOX13b & Potri.005G252800 & Chr05: 25454469-25456507 & AT4G35550 & 216 & 24.639 \\
\hline PtrWOX13c & Potri.002G008800 & Chr02: 484202-486345 & AT4G35550 & 215 & 24.479 \\
\hline
\end{tabular}

*Arabidopsis WOX genes that are closely classified with PtrWOXs according to the phylogenetic analysis of protein sequences. 


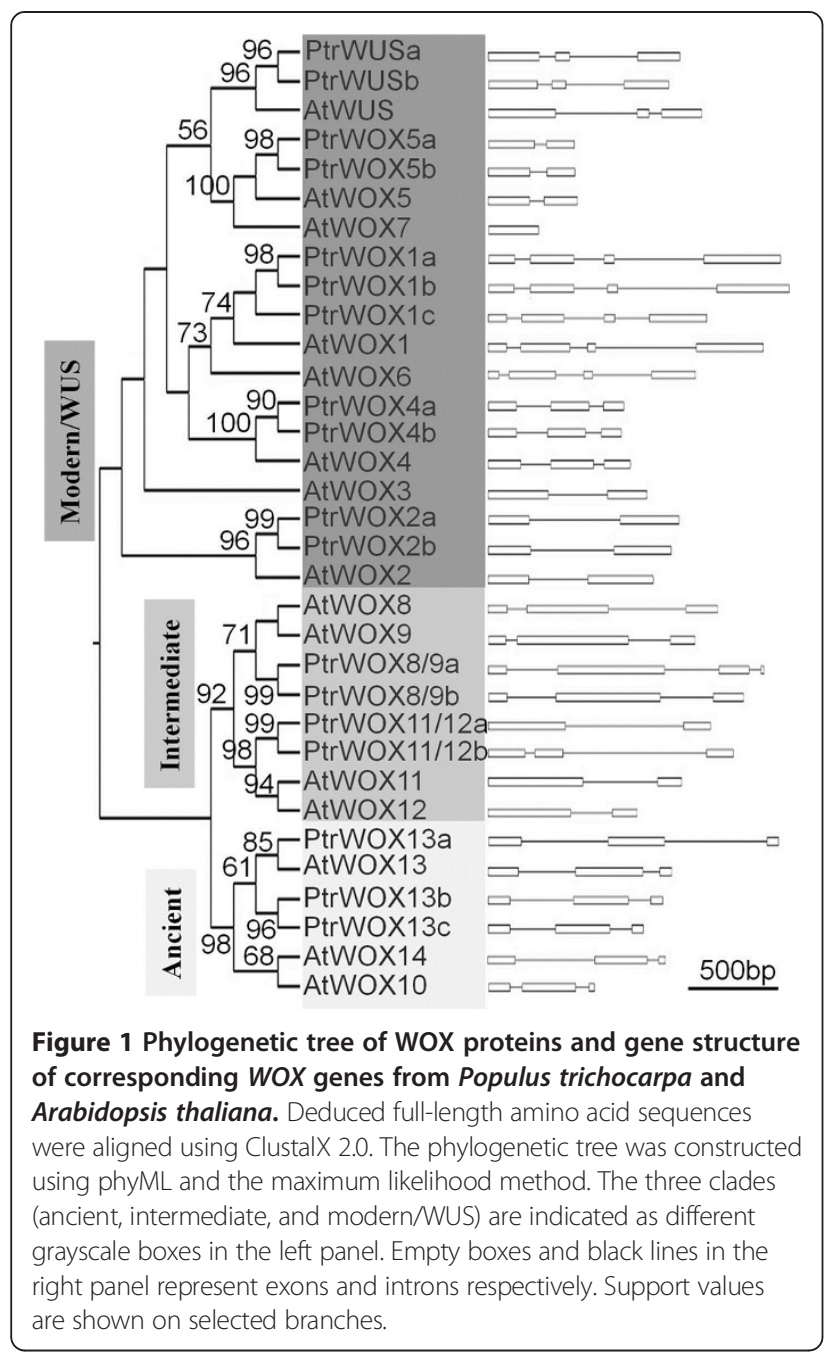

AtWOX10, AtWOX12, and AtWOX14 were missing from Vitis vinifera [28], Picea abies [30], and other woody plant species [28]. Details of these PtrWOX encoding genes, including their corresponding Arabidopsis counterparts, and amino acid lengths are listed in Table 1.

Using primers specific to each $\operatorname{Ptr} W O X$ gene (Additional file 2: Table S1), we identified and cloned 18 corresponding WOX genes from P. tomentosa (PtoWOXs). We found that PtoWOXs and PtrWOXs shared very high amino acid sequence similarities (86-99\%) and CDS sequence identities (Additional file 2: Table S2). Phylogenetic analysis revealed that the PtoWOXs could be classified identically to PtrWOXs (Figure 2).

\section{Chromosomal location and gene duplication of WOX genes in $P$. trichocarpa}

To better understand how WOX genes expanded in poplars, we took advantage of the sequenced genome of $P$. trichocarpa and mapped the chromosomal location of the 18 PtrWOX loci. These 18 PtrWOX sequences were

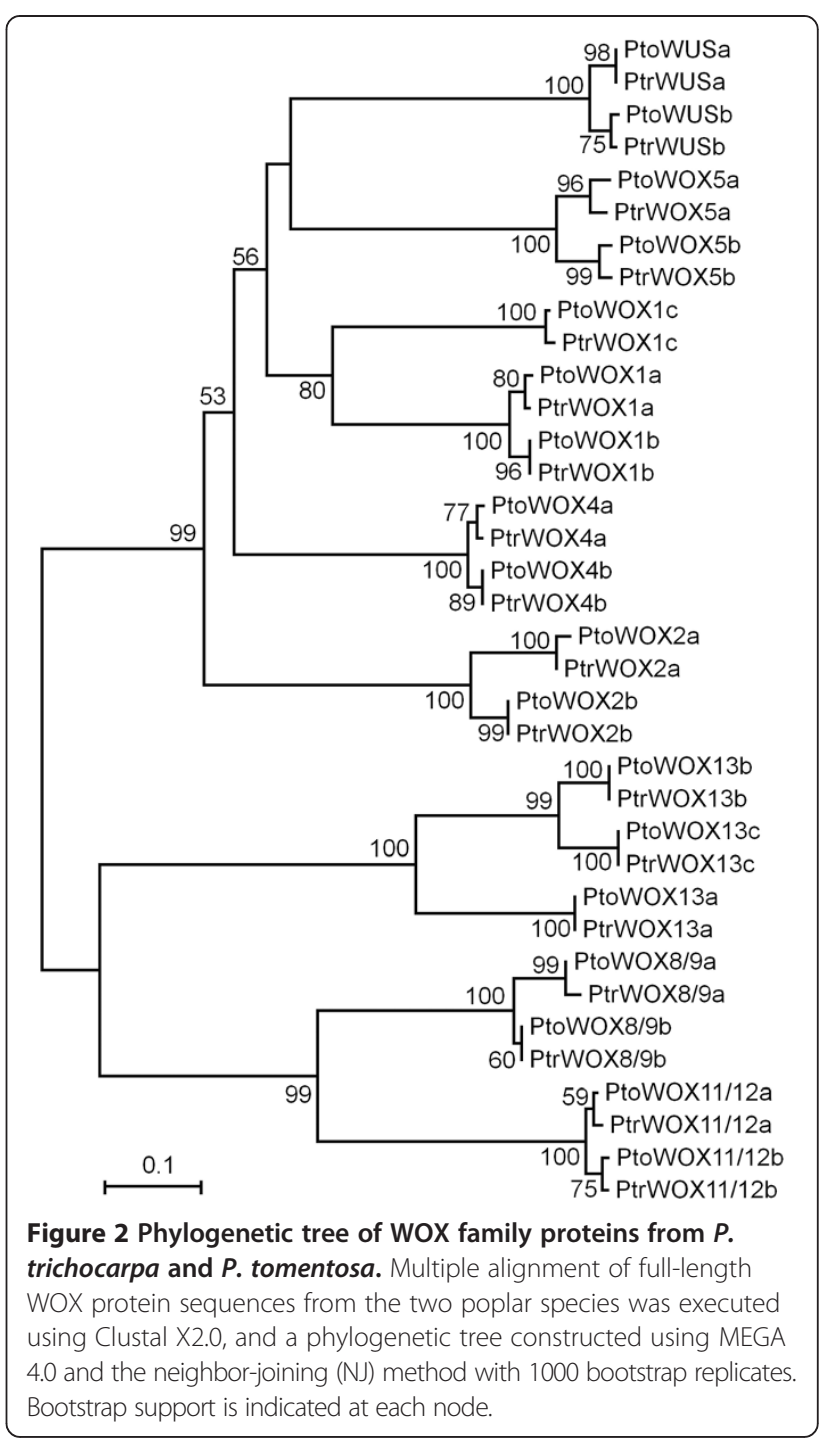

unevenly distributed among the 14 chromosomes, with the exception of chromosomes III, VI, XIII, XVI, and XVIII (Additional file 1: Figure S2). The chromosomal duplication map of $P$. trichocarpa was generated previously [31]. We identified five pairs of PtrWOX genes, PtrWOX1a-PtrWOX1b, PtrWOX4a-PtrWOX4b, PtrWOX5a-PtrWOX5b, PtrWOX11/12a-PtrWOX11/12b, and PtrWOX13b-PtrWOX13c in the duplicated segments of the $P$. trichocarpa genome. This suggests that expansion of the PtrWOX gene family was, at least partially, caused by chromosomal duplication events during the evolution of poplar species.

\section{Nuclear localization of PtoWOXs}

As a first step towards understanding the functions of WOXs in P. tomentosa, we examined the subcellular localization of PtoWUSa, PtoWOX4a, PtoWOX5a, PtoWOX11/12a, and PtoWOX13c members of selected 
PtoWOX subclasses. As transcription factors, WOXs should be targeted to the nucleus. To test this, we fused YFP at N-termini of PtoWUSa, PtoWOX4a, PtoWOX5a, and PtoWOX11/12a and the C-terminus of PtoWOX13c. In a transient expression assay of Nicotiana benthamiana leaf epidermal cells all fusion proteins were found in the nucleus (Figure 3).

\section{Diversified expression patterns of PtoWOX genes}

To gain insights into possible developmental and physiological functions of WOX proteins in P. tomentosa, the expression pattern of WOX genes in P. tomentosa Carr. were analyzed by semi-quantitative RT-PCR (Figure 4, Table 2). Two PtoWOX2s and two PtoWOX11/12 $s$ were mainly expressed in the roots. In contrast, PtoWUSb, and three PtoWOX1s were nearly absent from roots. Further to this, PtoWOX1a and $1 b$ were only expressed in leaves and PtoWOX1c only in the stem. PtoWOX5a and PtoWOX8/9a

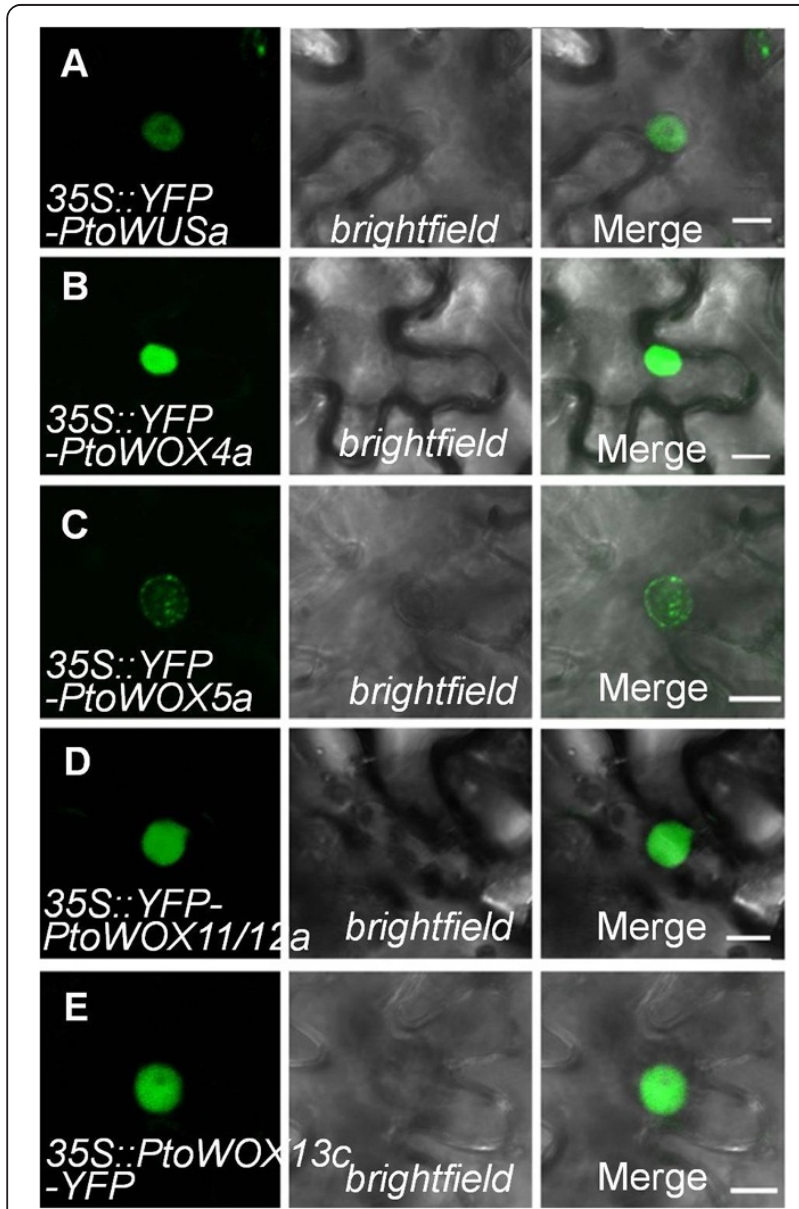

Figure 3 Subcellular localization of five PtoWoX proteins. The nuclear localization of PtoWUSa (A), PtoWOX4a (B), PtoWOX5a (C), PtoWOX11/12a (D), and PtoWOX13c (E) in a tobacco lower epidermal cell. The YFP channel, the bright field, and the merged images are shown on the left, middle, and right panels respectively. were highly expressed in both roots and leaves, while PtoWOX8/9b was expressed in both roots and stem. It is noteworthy that PtoWUSa, PtoWUSb, PtoWOX4a, and PtoWOX $4 b$ were strongly expressed in the CAM zone of poplar stems. In Arabidopsis, AtWUS is only expressed in the SAM [32]. In addition to the enrichment of PtoWUSa and PtoWUSb in the CAM zone, PtoWUSa was also expressed, albeit weakly, in roots, leaves, and stems. Similarly, AtWOX5 is restricted to the RAM [10], while PtoWOX5b was ubiquitously expressed. The only subclass of the ancient WOX clade in poplars, PtoWOX13s (PtoWOX13a, b, c), also exhibited a ubiquitous expression pattern. In a separate research project conducted in our laboratory, we generated a set of RNA-seq data for the hybrid poplar Populus alba X Populus glandulosa. Quantification of expression of the corresponding 18 WOX genes in P. alba X P. glandulosa (PagWOXs;

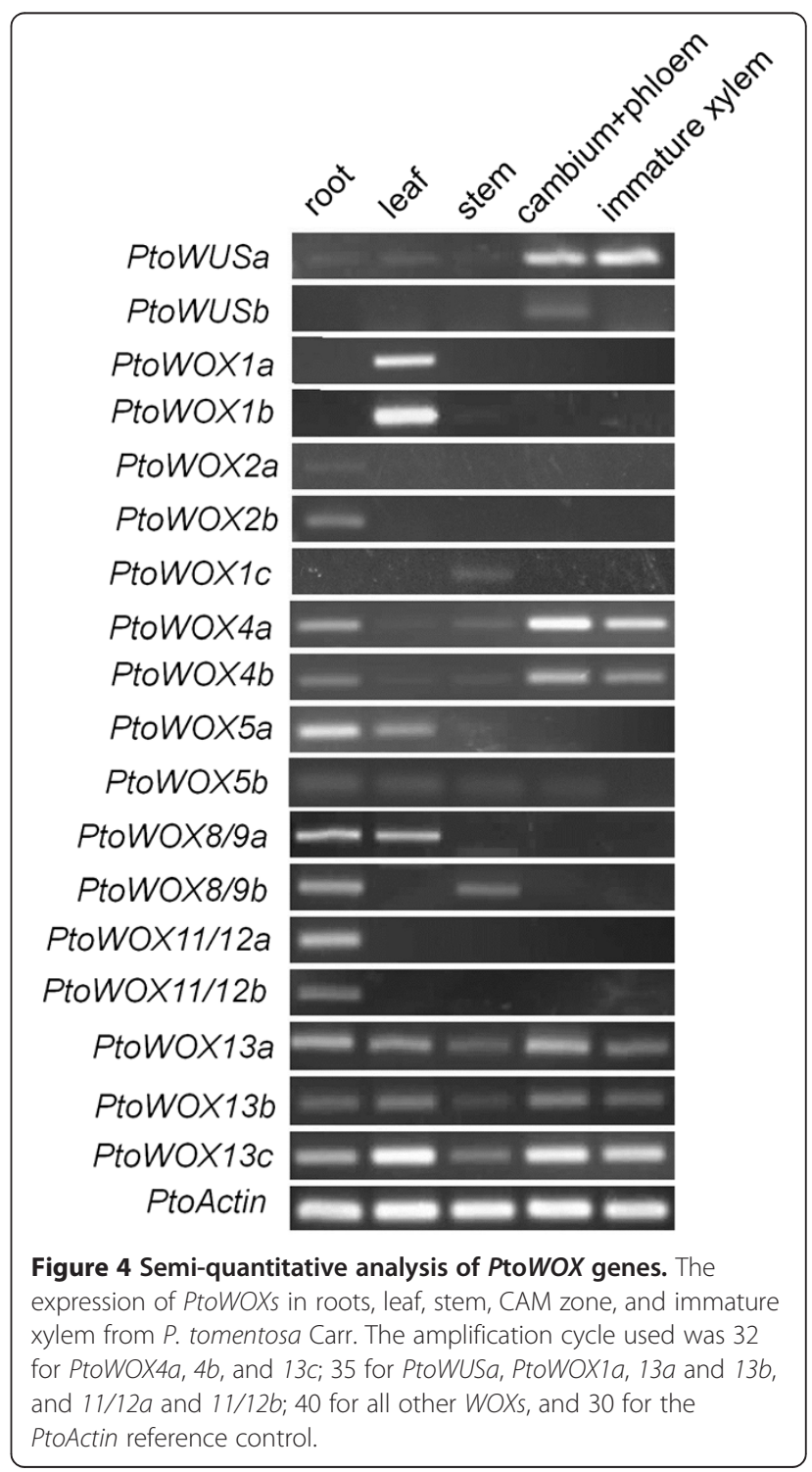


Table 2 Summary of the expression pattern of AtWOXs and PtoWOXs

\begin{tabular}{|c|c|c|c|c|}
\hline PtoWOXs & Expression pattern & AtPWOXs & Expression pattern & Ref \\
\hline PtoWUSa & Root, leaf, SAM, cambium, xylem & \multirow[t]{2}{*}{ AtWUS } & \multirow[t]{2}{*}{ SAM } & \multirow[t]{2}{*}[32]{} \\
\hline PtoWUSb & Cambium, xylem & & & \\
\hline PtoWOX1a & Leaf & \multirow[t]{3}{*}{ AtWOX1 } & \multirow{3}{*}{$\begin{array}{l}\text { Between the adaxial and abaxial } \\
\text { domains of leaf }\end{array}$} & \multirow[t]{3}{*}{33} \\
\hline PtoWOX1b & Leaf & & & \\
\hline PtoWOX1c & Stem & & & \\
\hline PtoWOX2a & Root & \multirow[t]{2}{*}{ AtWOX2 } & \multirow[t]{2}{*}{ Apical embryo domain } & \multirow[t]{2}{*}[34]{} \\
\hline PtoWOX2b & Root & & & \\
\hline \multicolumn{2}{|c|}{ No closely classified counterparts } & AtWOX3 & $\begin{array}{l}\text { flower primordia, floral organ primordia, } \\
\text { and young leaf primordia. }\end{array}$ & {$[35]$} \\
\hline PtoWOX4a & Root, Leaf, stem, cambium, xylem & AtWOX4 & \multirow{2}{*}{$\begin{array}{l}\text { Cambium, trichomes, stomata, phloem, } \\
\text { pericycle }\end{array}$} & \multirow[t]{2}{*}{ [12] } \\
\hline PtoWOX4b & & & & \\
\hline PtoWOX5a & RAM, leaf & \multirow[t]{2}{*}{ AtWOX5 } & \multirow[t]{2}{*}{ RAM } & \multirow[t]{2}{*}[10]{} \\
\hline PtoWOX5b & Root, leaf, stem & & & \\
\hline \multicolumn{2}{|c|}{ No closely classified counterparts } & AtWOX6 & Differentiating primordia and developing ovules & {$[16]$} \\
\hline \multicolumn{2}{|c|}{ No closely classified counterparts } & AtWOX7 & unknown & \\
\hline PtoWOX8/9a & Root, leaf & AtWOX8 & zygote, proembryo and embryo & [14] \\
\hline PtoWOX8/9b & Root, stem & AtWOX9 & $\begin{array}{l}\text { Developing embryos, proliferating tissues, SAM, } \\
\text { leaf primordia, floral meristems, epidermal layer } \\
\text { of the placenta and growing septum }\end{array}$ & [19] \\
\hline \multicolumn{2}{|c|}{ No closely classified counterparts } & AtWOX10 & \multicolumn{2}{|l|}{ unknow } \\
\hline PtoWOX11/12a & \multirow[t]{2}{*}{ Root } & OsWOX11 & \multirow{2}{*}{$\begin{array}{l}\text { Cell division regions in roots and shoots. } \\
\text { unknown }\end{array}$} & \multirow[t]{2}{*}[20]{} \\
\hline PtoWOX11/12b & & AtWOX11, 12 & & \\
\hline PtoWOX13a & \multirow[t]{3}{*}{ Root, leaf, SAM, cambium, xylem } & AtWOX13 & \multirow{3}{*}{$\begin{array}{l}\text { Root tip, emerging lateral roots, root, SAM } \\
\text { and leave vasculature, gynoecia }\end{array}$} & \multirow[t]{3}{*}[21]{} \\
\hline PtoWOX13b & & & & \\
\hline \multicolumn{2}{|l|}{ PtoWOX13C } & & & \\
\hline \multicolumn{2}{|c|}{ No closely classified counterparts } & AtWOX14 & Vascular tissue & {$[23]$} \\
\hline
\end{tabular}

The WOX expression patterns in other plant species were published in the corresponding references listed. Tissue names in italic indicate strong expression.

Additional file 1: Figure S3, Additional file 2: Table S3) indicated that the expression pattern of 18 PagWOX genes in the hybrid poplar was similar to that of the 18 PtoWOX genes in $P$. tomentosa, though there was a discrepancy between PtoWUSb and PagWUSb.

\section{Promoter::GUS based analysis of expression of PtoWOX genes}

RT-PCR is a rapid way to examine tissue specificity of gene expression, but it does not provide a good resolution. Since we are interested in the process of the root growth and wood formation in Populus tomentosa, a rootspecific PtoWOX11/12a, a root-enriched PtoWOX5a, and two generally expressed but CAM enriched PtoWOXs (PtoWUSa and PtoWOX4a) were selected for promoter:: GUS assays to examine their expressions in detail. Due to ease of genetic manipulation, the hybrid poplar $P$. alba $\mathrm{X} P$. glandulosa, a close relative of $P$. tomentosa, was chosen for gene transformation. In Arabidopsis, AtWOX5 is expressed specifically in the QC cells in the root tips
[10]. Semi-quantitative RT-PCR results revealed that in addition to being strongly expressed in the roots PtoWOX5a was weakly expressed in the leaves (Figure 4). The promoter::GUS assays indicated that PtoWOX5a was mainly expressed in a small area behind the cap region of ARs in the $P_{\text {PtoWOX5a: }}$ GUS transformed hybrid poplar (Figure 5B, see arrow). The PtoWOX5a promoter had a weak activity in the leaves (Figures 4 and $5 \mathrm{~A}$ ). Semi-quantitative RT-PCR identified PtoWOX11/12 $a$ as a root specific WOX gene in P. tomentosa (Figure 4). A GUS assay confirmed it was only expressed in the roots, especially in a small region behind the AR cap (Figure $5 \mathrm{C}$ and D, see arrow). Based on The semi-quantitative expression analysis revealed PtoWUSa and PtoPWOX4a were strongly expressed in the CAM zone, but were detectable in other examined tissues (Figure 4). GUS staining (Figure 5E-J) revealed that PtoWUSa and PtoPWOX4a were both strongly expressed in the CAM zone of stems (Figure $5 \mathrm{G}$ and $\mathrm{J}$ ); they were also weakly expressed in roots (Figure $5 \mathrm{~F}$ and I) as GUS 

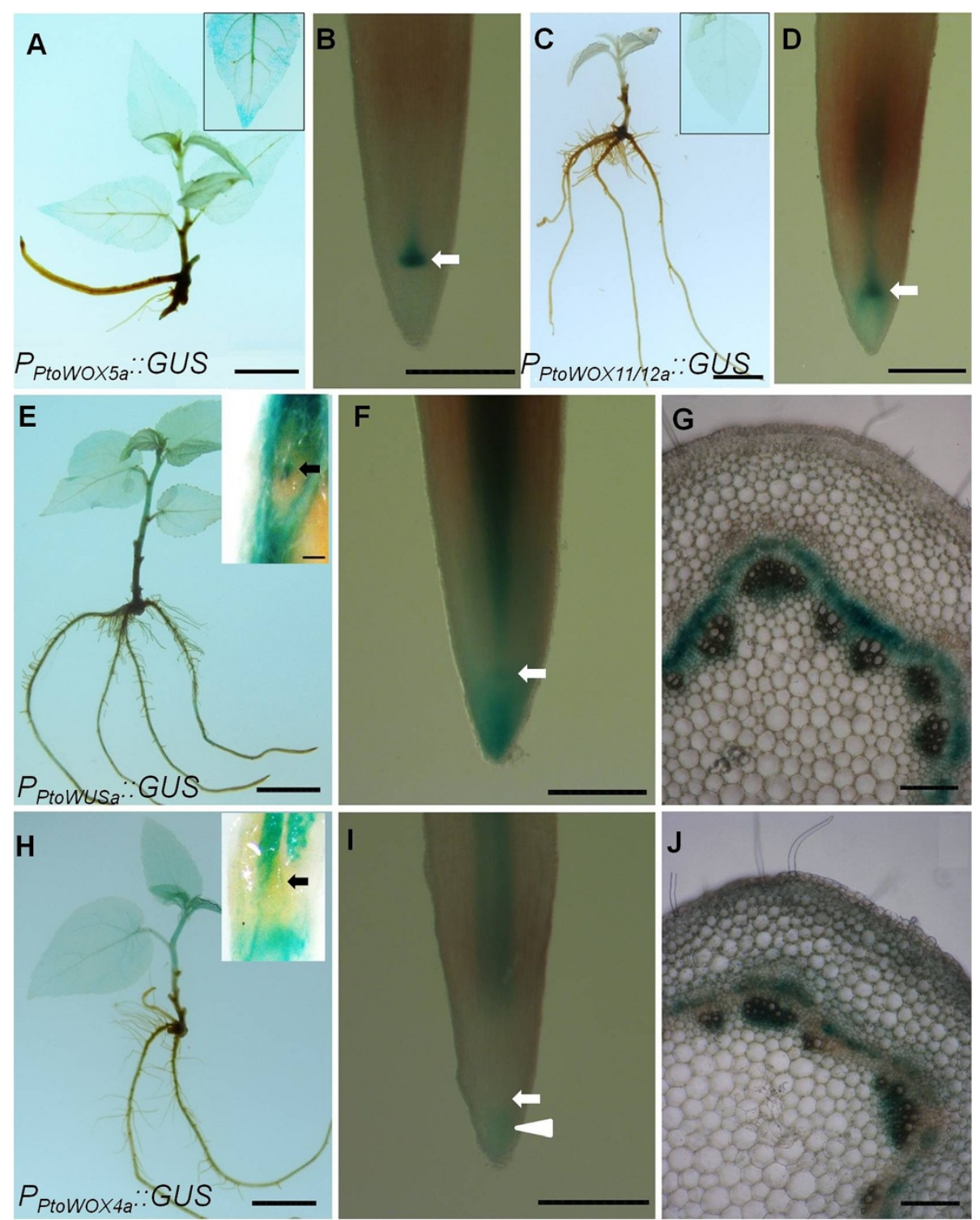

Figure 5 GUS-staining assays of four PtWOX promoters. (A-B) GUS expression in (A) 2-week-old sapling and (B) root of $P_{\text {PtowOX5a: }: G U S \text {-expressing }}$ poplar plants. The insert in A shows GUS expression in the leaf, and that the GUS expression in B is mainly focused on a small area immediately behind

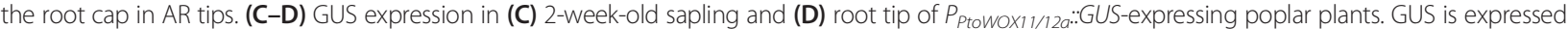
in a small area immediately behind the root cap in AR tips, and the insert in C shows no GUS expression in leaf. (E-G) GUS expression in (E) 2-week-old sapling, (F) root including a small area behind the root cap indicated by an arrow, and (G) the CAM zone of $P_{\text {Ptowusa: }}: G U S$-expressing poplar plants. The insert in E shows GUS expression in the SAM area in a halved shoot apical part. (H-J) GUS expression in (H) 2-week-old sapling, (I) root tip and (J) stem of $P_{\text {Ptowox4a: }}$ GUS-expressing poplar plants. The insert in H shows no GUS expression in the SAM area in a halved shoot apical part. The bar represents

$1 \mathrm{~cm}$ in $\mathbf{A}, \mathbf{C}, \mathbf{E}$, and $\mathbf{H} ; 1 \mathrm{~mm}$ in $\mathbf{D} ; 0.5 \mathrm{~mm}$ in $\mathbf{B}, \mathbf{F}$, and $\mathbf{I}$; and $0.1 \mathrm{~mm}$ in $\mathbf{G}, \mathbf{J}$, and the inset of $\mathbf{E}$.

staining of roots of transgenic lines expressing either $P_{P t o-}$ WUSa: $: G U S$ or $P_{\text {PtoWOX4a: }: \text { GUS occurred after a prolonged }}$ period. Interestingly, in the $P_{\text {PtoWUSa: }}$ GUS lines, a small region behind the root cap could be stained (Figure 5F, arrow), though activity of $P_{P t o w U S a}: G U S$ was not limited to this small region in roots. In $P_{P t o W O X 4 a}: G U S$ transgenic lines, the small region behind the root cap region was not stained, but the root cap region of ARs could be stained (Figure 5I, arrowhead).
Dynamic expression of PtoWOXs during the regeneration of adventitious shoots and roots in $P$. tomentosa

The regeneration of adventitious shoot (AS) and AR using leaf or stem explants is one of the best tools for rapid propagation and genetic manipulation of poplars. These regeneration processes involve the re-establishment of SAM and RAM, which leads to the final differentiation of leaves and roots. Therefore regeneration of AS and AR is an excellent system for studying stem 
cell initiation, cell fate determination, and hormonal signaling [36]. AS regeneration is composed of several successive stages, including the $\mathrm{B} 1$ pre-induction stage (Additional file 1: Figure S4A), the B2 callus induction stage (Additional file 1: Figure S4B), the B3 callus expansion stage (Additional file 1: Figure S4C), the B4 callus transition stage (Additional file 1: Figure S4D), the B5 AS formation stage (Additional file 1: Figure S4E), and the B6 AS growth stage (Additional file 1: Figure S4F). To investigate whether PtoWOXs play roles in the regeneration of AS we examined the dynamics of PtoWOX expression using quantitative real-time PCR. Among the 18 PtoWOXs, 10 had significant changes in expression during AS regeneration (Figure 6A). The expression levels of PtoWUSa, PtoWOX4a, PtoWOX4b, PtoWOX5a, and PtoWOX5b changed over twenty-fold during the regeneration of AS. They had different expression dynamics with maximum expression of PtoWOX4a/b seen at earlier stages (B2/B3), and PtoWOX5a/b at later stages (B4) (Figure 6). All three PtoWOX13s were expressed at a relatively low level in AS regeneration but were slightly induced during the AS regeneration process (Figure 6A). In contrast, expression of PtoWOX1a and $1 b$ was slightly down-regulated in AS regeneration. This down-regulation of PtoWOX1s may be a technical artifact as both PtoWOX1s were expressed specifically in leaves, thus their expression in the B1 stage may be still at a high level. Nevertheless, we view this as an indication that PtoWOX1s are not involved in SAM and AS regeneration. Finally, transcription of PtoWOX2a, PtoWOX2b, PtoWOX11/12a, and PtoWOX11/12b, whose expression was mainly in the roots, was unchanged during AS regeneration.
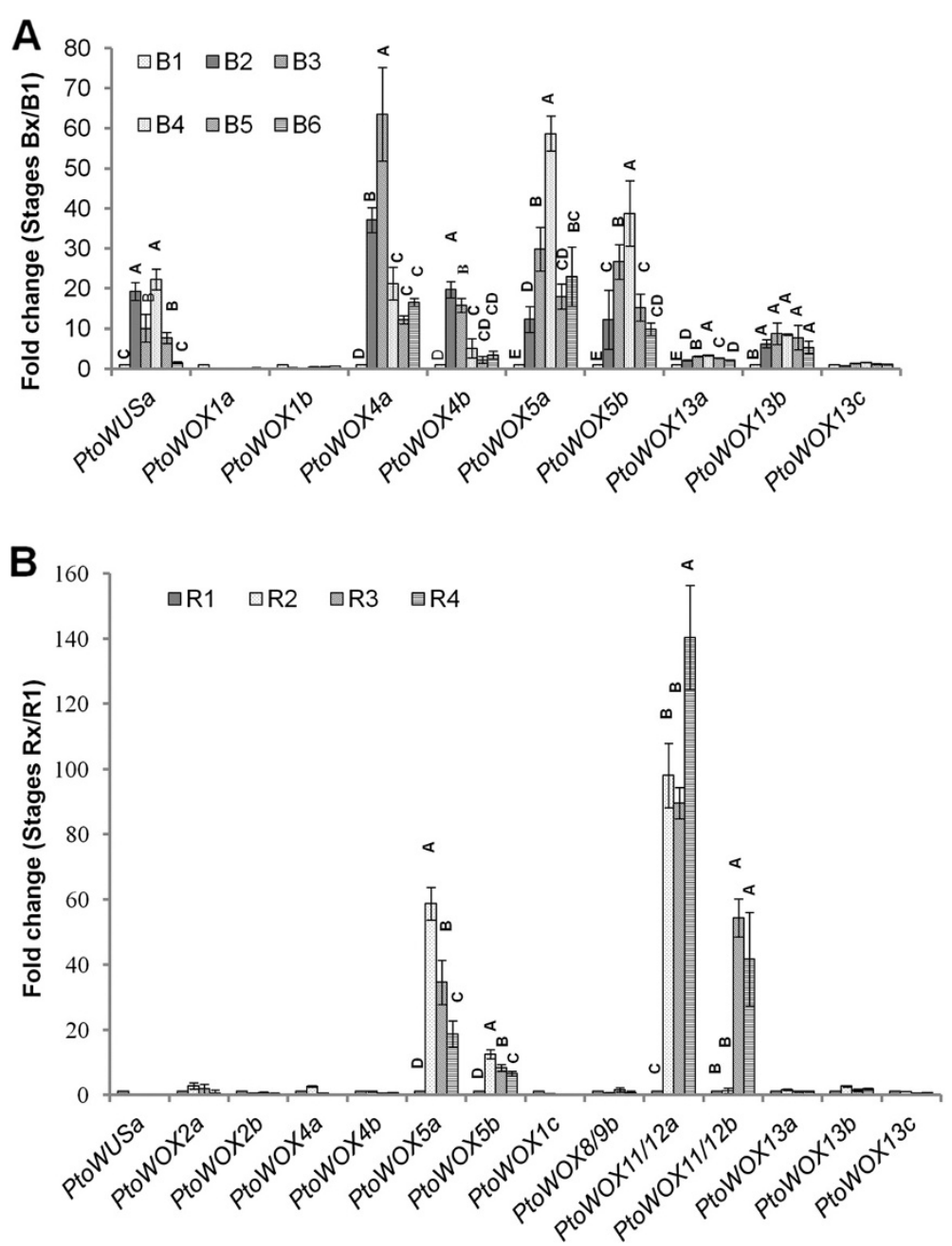

Figure 6 Quantitative PCR analysis of dynamic expression of PtoWOXs in the regeneration of AS and AR. (A) Dynamic changes of PtoWOXs in AS regeneration. The expression of PtoWUSa, PtoWOX4a, 4b, 5a, and $5 b$ is significantly changed. B1-B6 are six chronological stages during AS regeneration. (B) Dynamic changes of PtoWOXs in AR regeneration. PtoWOX5a, 5b, 11/12a, and 11/12b are significantly changed. R1-R4 are four chronological stages during AR regeneration. Insignificant differences, according to LSD test $(P<0.01)$, are denoted using the same letters. Error bars represent standard deviation. 
AR regeneration is composed of a $\mathrm{R} 1$ pre-induction stage (Additional file 1: Figure S4G), R2 callus formation stage (Additional file 1: Figure S4H), R3 AR emergence stage (Additional file 1: Figure S4I), and a R4 AR elongation stage (Additional file 1: Figure S4J). We examined the dynamics of PtoWOXs expression to gain insights into their possible roles in these processes. Expression of 14 out of 18 PtoWOXs was detected and changed during the AR regeneration process (Figure 6B). Among these, expression of four WOX genes: PtoWOX5a, PtoWOX5b, PtoWOX11/12a, and PtoWOX11/12b changed significantly during AR regeneration (Figure $6 \mathrm{~B}$ ). The expression of PtoWOX11/12a was over 80 -fold stronger and maintained a high level in the R2 though R4 stages. PtoWOX11/12b was induced at the R3 and R4 stages, but not at the R1 or R2 stage. It is interesting that although expression of PtoWOX5a and PtoWOX5b was induced at the R2 stage, their expression gradually decreased in the following stages. Similarly, root specific WOXs were not induced in AS regeneration; the expression levels of PtoWOX1a and PtoWOX1b, two leaf-specific WOX genes in P. tomentosa (Figure 4), were not changed.

\section{Ectopic expression of PtoWOX5a and PtoWOX11/12a as along with PtoWUSa and PtoWOX4a promotes AR regeneration}

Because of the high expression of PtoWOX5s, PtoWOX11/ 12 s, PtoWUSa, and PtoWOX4a in the roots, and the strong induction of PtoWOX5s and PtoWOX11/12 $s$ in the regeneration of $A R$, we were interested in the roles they play in the AR regeneration process. We used a $35 \mathrm{~S}$ promoter to ectopically express PtoWOX5a and PtoWOX11/12a along with PtoWUSa and PtoWOX4a in the hybrid poplar ( $P$. alba X P. glandulosa). AR regenerated in stem segments of three independent transgenic plants were quantified at the young sapling stage. All four ectopically expressed WOX genes promoted AR regeneration in the transgenic hybrid poplar lines (Figure 7). It appeared that PtoWOX5a and PtoWOX11/12a had a better ability than PtoWUSa and PtoWOX4a to promote the regeneration of AR. This is consistent with the strong induction of PtoWOX5a and PtoWOX11/12a in the regeneration of AR. Although the expression of PtoWUSa and PtoWOX4a was not obviously induced (Figure 6B), their effect on the regeneration of ARs when ectopically expressed indicated that the four PtoWOX genes may have overlapping functions.

\section{Discussion}

\section{Diversification of WOX genes in $P$. trichocarpa and $P$. tomentosa}

We identified 18 WOX encoding genes in both $P$. trichocarpa and $P$. tomentosa. Although these poplar WOX genes could be categorized into modern/WUS, intermediate, and ancient clades, it is interesting that no WOX genes from $P$. trichocarpa and $P$. tomentosa are classified together with AtWOX3, AtWOX6, AtWOX7, $A t W O X 10$, or AtWOX14. Furthermore, many of the WOX genes in $P$. trichocarpa had sister copies owing to chromosomal duplication events.

While the roles of $A t W O X 6$ and $A t W O X 7$ are not well defined, $A t W O X 3$ is expressed in leaves and involved in leaf blade outgrowth [15,37]. Moreover, the function of the AtWOX3 protein can be fully complemented by AtWUS [15], and partially complemented by AtWOX4 [17]. It is possible that the WOX3-mediated function of leaf blade outgrowth regulation is compensated by other modern WOX members in poplars. In this regard, we note that the PtoWOX1a gene and PtoWOX1b gene are specifically expressed in $P$. tomentosa leaves (Figure 4).

Arabidopsis contains three ancient WOX genes, AtWOX10, AtWOX13, and AtWOX14. P. trichocarpa and P. tomentosa also have three ancient WOX genes, PtrWOX13a, b, and $c$; these are very similar in sequence, with PtrWOX13b and PtrWOX13c being sister pairs. Grape also has three ancient WOX genes, $V \nu W O X 13 A, B$, and $C$. [28]. There appears to be functional diversification in $A t W O X 10, A t W O X 13$, and AtWOX14; the AtWOX13 gene is involved in replum formation during fruit development [21], AtWOX14 acts redundantly with AtWOX4 in vascular cell division [23], while the role of $A t W O X 10$ is unknown. The three ancient WOX genes of $P$. trichocarpa and $P$. tomentosa are ubiquitously expressed in all examined tissue in poplars (Figure 4). It is possible that the diversified functions of ancient AtWOX10, 13, and 14 in Arabidopsis may be maintained by three ancient WOX genes, namely WOX $13 a, b$, and $c$ in poplars.

\section{Expression of PtoWOX genes}

Protein function can be specified in terms of temporally and spatially regulated gene expression. We note that some PtoWOXs genes are expressed differently from their Arabidopsis counterparts (Table 2). AtWUS is expressed specifically in the rib-meristem (RM) cells beneath the central zone of the SAM, and it maintains the stem cell population via a regulatory loop with CLAVATA genes in Arabidopsis [9,32]. In poplar, the expression of PtoWUSa is not limited to the SAM and is strongly induced in the regeneration of AS; it is also detected in roots, stem and leaves (Figure 4, Figure 5E-G), and particularly in the CAM zone of stems. A major difference between Arabidopsis and poplar is that the latter is a perennial plant with annual differentiation of vascular tissues from CAM. It seems that the PtoWUSa gene may also be involved in vascular tissue differentiation. In Arabidopsis, the AtWOX4 gene is required for vascular differentiation from CAM cells [12,38]. Both PtoWOX4a and PtoWOX4b genes are strongly expressed in CAM cells 

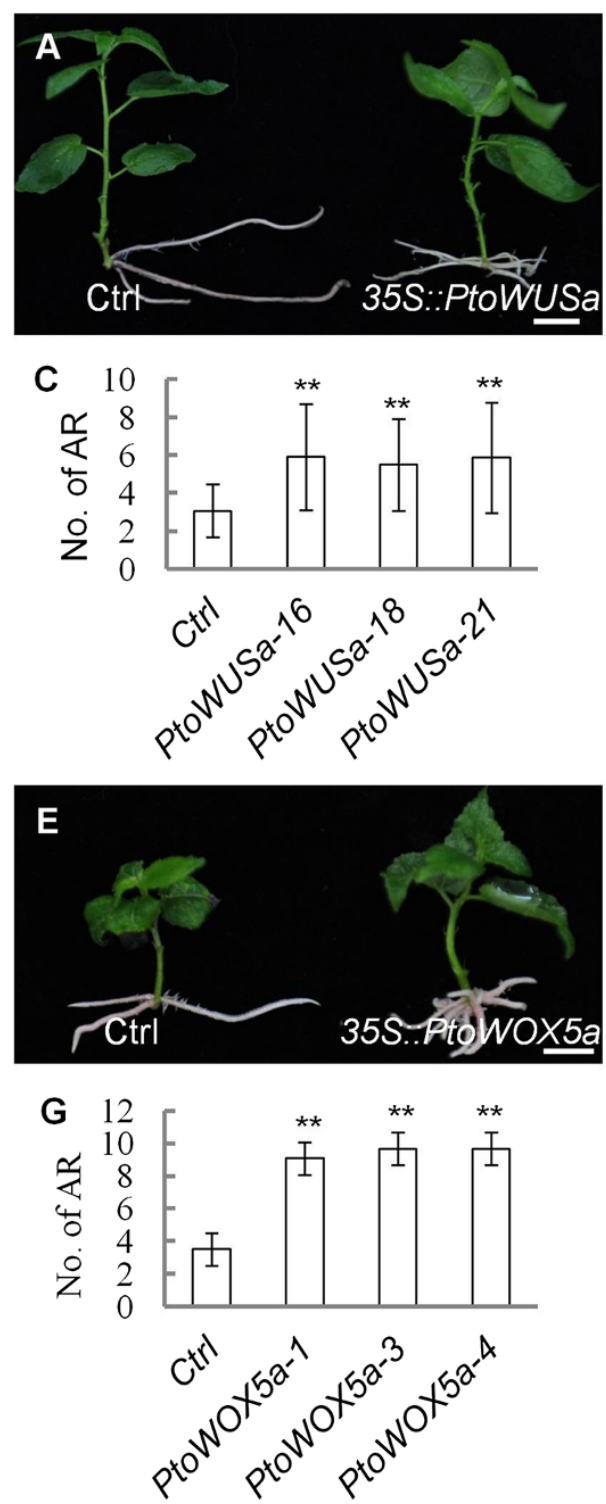
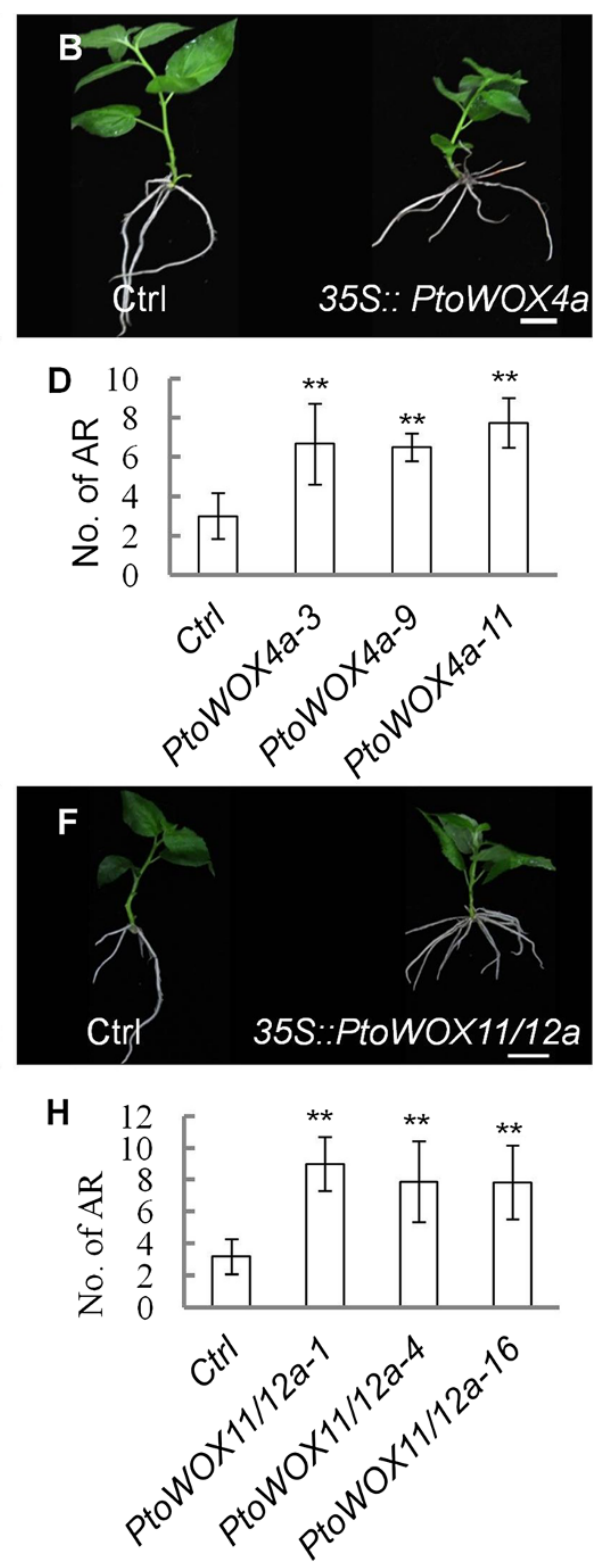

Figure 7 Ectopic over-expression of four PtoWOXs promotes regeneration of AR. (A-B, E, and F) Regenerated ARs in transgenic poplar plants ectopically expressing (A) PtoWUSa, (B) PtoWOX4a, (E) PtoWOX5a, and (F) PtoWOX11/12a. Wild type and over-expressing lines are on the left and right of each panel, respectively. (C-D, G, and H) Statistical result of ARs in different transgenic lines. No. of AR: adventitious root numbers; bars in $\mathbf{A}, \mathbf{B}, \mathbf{E}$, and $\mathbf{F}$ represent $1 \mathrm{~cm}$. ${ }^{*}$ in $\mathbf{C}, \mathbf{D}, \mathbf{G}$, and $\mathbf{H}$ indicates significant difference using LSD test at $P \leq 0.01$.

and are strongly induced in AS regeneration, but not in AR regeneration (Figure 6). Therefore, differently from PtoWUSa, the function of the PtoWOX4 gene in CAM cells may be maintained in poplars. It will be interesting to test whether PtoWUSa and PtoWOX4 act differently in vascular tissue differentiation in poplars.

AtWOX5 is a QC cell specific gene in Arabidopsis [11]. It seems that functional expansion also occurs for PtoWOX5s. In poplars, the PtoWOX5a gene is strongly expressed in a small region that resembles $\mathrm{QC}$ cells, but is also detectable in young leaves (Figure 4). Furthermore, PtoWOX5a expression is strongly induced in the regeneration of AR as well as in the AS. Therefore, we speculate that the function of PtoWOX5a and PtoWOX5b may have expanded from roots to leaves in poplars.

Although the expression pattern of AtWOX11 in Arabidopsis has not been reported, expression of Os WOX11 in rice is detected in cell division regions of both roots and shoots [20]. We note that expression of both PtoWOX11/12 $s$ is restricted to a small area behind the root cap region that resembles the root QC in AR tips, and probably the pericycle (Figure 5D). Thus, the action 
of PtoWOX11/12 s may be restricted to root development in poplars. During the regeneration of AR, the expression levels of PtoWOX11/12 s and PtoWOX5s are all strongly induced; however, it is interesting that the expression of PtoWOX11/12a is induced at the R2 stage, while the expression of PtoWOX11/12b is induced at the R3 stage. It is likely that PtoWOX11/12a acts differently from PtoWOX11/12b in the regeneration of AR.

\section{Four PtoWOX proteins can perform similar functions in promoting AR regeneration}

A key function of WOX proteins is to maintain the stem cell population in different tissues and organs $[9,11,12,39]$. Recently, it has been demonstrated [8] by using a lam1 complementation screen of WOX proteins in $N$. sylvestris that modern members of WOX proteins may have acquired additional functions during evolution that lead to functional specificity of this clade in the regulation of leaf blade outgrowth. In this study, we found that when ectopically expressed, three members of the modern WOXs (PtoWOX4a, PtoWOX5a, and PtoWUSa) and a member of the intermediate WOXs (PtoWOX11/12a) promote AR regeneration (Figure 7 ). In our promoter:: GUS based expression analysis, the PtoWUSa, PtoWOX5a, and PtoWOX11/12a genes are expressed in a small region in the root tips resembling QC cells, the PtoWOX4a gene however, was not expressed in restricted areas in the root tips. Therefore, it is likely that members of the modern and intermediate WOXs can perform similar functions in the regeneration of AR in poplars, even if the gene is not normally expressed in root tip cells.

There is a clear divergence in the amino acid sequences of the 15 Arabidopsis and 18 poplar WOX proteins, with only modern WOX proteins possessing a WUS-box domain. Lin et al. [8] demonstrated that the WUS-box is required for the regulation of leaf blade outgrowth. Many different WOXs act in different cells and tissues to maintain the stem cell population $[9,11,12,39]$, thus a common action mechanism for WOX proteins in maintaining the stem cell niche has been proposed [8]. Our results add support to this viewpoint. The regeneration of AR relies on a balance between cell differentiation and renewal of stem cells in the RAM [11]. It is possible that PtoWOX5a and PtoWOX11/12a, whose genes are expressed and strongly induced in the regeneration of $\mathrm{AR}$, are major players in maintaining the stem cell niche in root tips. However, the function of PtoWOX5a and PtoWOX11/12a in maintaining the stem cell niche in root tips may be partially overlapped by PtoWUSa or PtoWOX4a. When the latter two PtoWOX genes are ectopically expressed they promote the regeneration of AR. This functional overlapping may be attributed to a common mechanism for maintaining the stem cell niche. It is possible that a WOX domain other than the
WUS-box is responsible for this common action. It will be interesting to examine which WOX domains have evolutionary significance in the function of WOXs in stem cell maintenance.

\section{Conclusions}

This study represents a step forward in our understanding of the functions and mechanisms of 18 WOXs found in poplar, in particular, their possible roles in vascular as well as root development of poplars. Based on the expression of PtoWOX genes revealed in this study, it is attempted to suggest that, in poplars, PtoWOX4s and PtoWUSs are involved in vascular development, while PtoWOX5s and ProWOX11/12s are major players in root development. Despite these possible functional specificities, it appears that there is a common action mechanism for different PtoWOXs in maintaining different stem cell niches.

\section{Methods}

\section{Bioinformatic analysis}

The $P$. trichocarpa genome (release 3.0, http://www. phytozome.net/poplar) was blasted using AtWOXs protein sequences as queries with NCBI BLASTP. Obtained sequences were used as secondary queries to re-blast the $P$. trichocarpa genome. After removing redundant sequences, multiple alignments of full length sequences of AtWOX and PtrWOX proteins were performed using the Clustal X2.0 program [40]. Maximum likelihood (ML) phylogenetic trees were constructed using PhyML (v3.0) with JTT amino acid substitution model, 1000 bootstrap replicates, estimated proportions of invariable sites, estimated gamma distribution parameters, and an optimized starting BIONJ tree [41,42]. A multiple alignment of full-length WOX protein sequences from $P$. trichocarpa and $P$. tomentosa was executed using Clustal X2.0 [40], and a phylogenetic tree constructed using MEGA 4.0 by the neighbor-joining (NJ) method with 1000 bootstrap replicates. The trees have been submitted to Treebase under study number 15612 (http://treebase.org/treebase-web/search/study/ summary.html?id=15612). Accession numbers used in this study are listed in Additional file 2: Table S4. Exon and intron structures of individual PtrWOXs were illustrated using the Gene Structure Display Server (GSDS, http://gsds.cbi.pku.edu.cn/) [43] by aligning the cDNA sequences with the corresponding genomic DNA sequences from www.phytozome.net. All 18 PtrWOX genes were mapped to $P$. trichocarpa chromosomes. Whole-genome duplication analyses were accomplished as described in Tuskan et al. [30]. The MEME program (version 4.3.0, http://meme.sdsc.edu) [44] was used for elucidation of motifs in HDs. MEME was run locally with the following parameters: number of repetitions - any; maximum number of motifs - 20; and the optimum motif widths were constrained from 6 to 21 residues. 


\section{Plasmids and constructs}

The coding sequences of all PtoWOXs except for PtoWUSb (submitted to NCBI by another group) were amplified from the cDNA of $P$. tomentosa, and cloned into pDNOR222.1 (Life technologies, Carlsbad, California, U.S.) (to produce $p E N T R s$ ) for sequencing. PtoWUSa, PtoWOX4a, PtoWOX5a, and PtoWOX11/12a were subcloned into pMDC32 to produce 35S::PtoWUSa, 35S:: PtoWOX4a, 35S::PtoWOX5a, and 35S::PtoWOX11/12a constructs. The resultant $p E N T R$ constructs were then recombined into pEarleyGate104 (ABRC stock DB3-686) to produce 35S::YFP-PtoWUSa, 35S::YFP-PtoWOX4a, 35S::YFP-PtoWOX5a, and 35S::YFP-PtoWOX11/12a constructs using the Gateway cloning system (Life technologies, Carlsbad, California, U.S.). PtoWOX13c without the stop codon was amplified and subcloned into pEarleyGate101 (ABRC stock DB3-683) to produce 35S::Pto WOX13c-YFP. The primer sequences used for amplification of PtoWOXs are listed in Additional file 2: Table S1. 5'UTR fragments, $2 \sim 3 \mathrm{~kb}$ in size, of PtoWUSa, PtoWOX4a, PtoWOX5a, and PtoWOX11/12a were amplified from the genomic DNA of $P$. tomentosa Carr. Primer sequences and promoter lengths are listed in Additional file 2: Table S1. The amplified promoter fragments were cloned into pDNOR222.1, and then subcloned into pMDC164 to

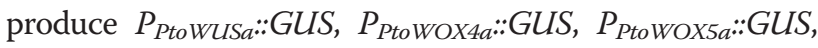
and $P_{\text {PtoWOX11/12a: }}$ GUS constructs using the gateway cloning system (Life technologies, Carlsbad, California, U.S.). At least three independent lines were used for analysis.

\section{Plant cultivation and transformation}

Tobacco plants (Nicotiana benthamiana) used for transient expression were grown on soil under an 8/16 h (day/night) photoperiod at $20^{\circ} \mathrm{C}$. All constructs were introduced into Agrobacterium tumefaciens strain GV3101 by electroporation. A single A. tumefaciens colony containing 35S::YFP-PtoWUSa, 35S::YFP-PtoWOX4a, 35S:: YFP-PtoWOX5a, 35S::YFP-PtoWOX11/12a, or 35S:: PtoWOX13c-YFP was used to inoculate $2 \mathrm{~mL}$ of YEP medium (per liter: $10 \mathrm{~g}$ tryptone, $10 \mathrm{~g}$ yeast extract, $5 \mathrm{~g} \mathrm{NaCl}, \mathrm{pH}$ 7.0), supplemented with $50 \mathrm{mg} / \mathrm{L}$ kanamycin, $10 \mathrm{mg} / \mathrm{L}$ gentamycin, and $34 \mathrm{mg} / \mathrm{L}$ rifampicin. Bacterial cultures were incubated at $28^{\circ} \mathrm{C}$ with agitation until $\mathrm{OD}_{600}=0.5$. $0.5 \mathrm{~mL}$ of culture was transferred into an Eppendorf tube, and the bacteria pelleted by centrifugation at $2000 \times g$ for $5 \mathrm{~min}$ in a microcentrifuge at room temperature. The pellet was washed twice with $0.5 \mathrm{~mL}$ of infiltration buffer (10 mM $\mathrm{MgCl}_{2}, 150 \mu \mathrm{M}$ acetosyringone) and resuspended in $0.5 \mathrm{~mL}$ of the same buffer. The inoculum concentration of 35S::YFP-PtoWUSa, 35S::YFPPtoWOX4a, 35S::YF-PtoWOX5a, 35S::YFP-PtoWOX11/12a, and 35S::PtoWOX13c-YFP $\left(\mathrm{OD}_{600}=0.1\right)$ was adjusted by diluting the bacterial suspension with the infiltration buffer. The inoculum was delivered to tobacco lower epidermal leaf cells by gentle pressure infiltration using a 1-mL syringe without a needle. Following infiltration, plants were incubated under dark conditions for $6 \mathrm{~h}$, and then grown under normal conditions, as described above. Tobacco leaves were analyzed 3 days after infiltration.

Hybrid poplar ( $P$. alba X P. glandulosa) clone $84 \mathrm{~K}$ used for transformation were kept at $23-25^{\circ} \mathrm{C}$ under a $16 / 8 \mathrm{~h}$ (day/night) photoperiod, with light intensity of $50 \mu \mathrm{M} \mathrm{m}^{-2} \mathrm{~s}^{-1}$ provided by cool white fluorescent tubes. Leaf-discs from $84 \mathrm{~K}$ were infected with Agrobacterium cultures containing $P_{\text {PtoWUSa }}:$ GUS, $P_{\text {PtoWOX4a }}:$ GUS, $P_{\text {PtoWOX5a: }}: G U S, P_{\text {PtoWOX11/12a: }}: G U S, 35 S:: P t o W U S a, 35 S::$ PtoWOX4a, 35S::PtoWOX5a, and 35S:: PtoWOX11/12a constructs with $\mathrm{OD}_{600}=0.3-0.8$. Infected leaf-discs were co-cultured with Agrobacteria in the AS induction medium [SIM; Murashige-Skoog (MS) basal medium with $0.5 \mathrm{mg} / \mathrm{l}$ 6-benzyl aminopurine (6-BA) and $0.05 \mathrm{mg} / \mathrm{l}$ naphthaleneacetic acid (NAA)] in the dark for 3 days at $22 \pm 2^{\circ} \mathrm{C}$. Leaf-discs were transferred on SIM with $3 \mathrm{mg} / \mathrm{L}$ hygromycin and $200 \mathrm{mg} / \mathrm{L}$ timentin under a $16 \mathrm{~h} / 8 \mathrm{~h}$ (light/dark) regime. After one month, individual regenerated shoots were removed and transferred onto root induction medium (RIM, 1/2 MS medium supplemented with $0.05 \mathrm{mg} / \mathrm{L} \mathrm{IBA}, 0.02 \mathrm{mg} / \mathrm{L} \mathrm{NAA}$ ) containing $3 \mathrm{mg} / \mathrm{L}$ hygromycin and $200 \mathrm{mg} / \mathrm{L}$ timentin for AR induction. For AS induction, leaf discs were cultured on SIM for 18 days (Additional file 1: Figure S6A-F), while stems were cultured on RIM for 9 days for AR regeneration (Additional file 1: Figure S6G-J). At least three independently transformed lines were used for overexpression analysis and promoter::GUS assay analysis. At least 25 clones of each overexpressed line were used for AR regeneration. All experiments were repeated at least three times with similar results. The regeneration of AS and AR in $P$. tomentosa was performed as above, but without the Agrobacteria co-cultured procedure.

\section{RNA isolation, RT-PCR, qRT-PCR, and RNA-seq analysis}

Total RNAs were extracted from roots, leaves, stem, CAM zone, and immature xylem of $P$. tomentosa Carr, and from materials at different stages and vegetative tissues of the hybrid poplar $P$. alba X P. glandulosa for RNA-sequencing using an RNeasy Plant Mini Kit and RNase-free DNase I set (Qiagen, Hilden, Germany). First-strand cDNA synthesis was carried out with approximately $1.5 \mu \mathrm{g}$ RNA using the SuperScript III first-strand synthesis system (Life technologies, Carlsbad, California, U.S.) according to the manufacturer's instructions. Specific RT-PCR primers were designed to have melting temperatures of $58-60^{\circ} \mathrm{C}$ and amplicon lengths of 150-260 bp using Primer3 software (http://frodo.wi. mit.edu/primer3/input.htm). The amplified fragments were separated on agarose gel electrophoresis. Real-time qRT-PCR was performed in quadruplicate using the SYBR 
Premix Ex Taq ${ }^{\text {Tw }}$ II Kit (TaKaRa Dalian, Dalian, China) on a Roche lightCycler 480 (Roche Applied Science, Penzberg, Upper bavaria, Germany) according to the manufacturer's instructions. Quantification was performed using Lightcycler 480 software (Version 1.5.1.62, Roche). Expression was normalized relative to the control (Actin) using Roche LightCycler advanced relative quantification analysis (E-method, http://www.roche-applied-science.com/shop/products/gene-quantification-on-the-lightcycler-480-system) and fold changes (relatively to B1 or R1 stage) are shown in Figure 6. All experiments were repeated at least three times with similar results.

\section{Promoter::GUS assay}

Histochemical GUS staining was performed as follows: 2-week old seedlings and 4-week old stem sections were first fixed in $90 \%$ cold acetone. Each sample was washed three times on ice using GUS staining buffer containing $50 \mathrm{mM}$ sodium phosphate (pH7.0), $2 \mathrm{mM}$ potassium ferrocyanide, $2 \mathrm{mM}$ potassium ferricyanide, $10 \mathrm{mM}$ EDTA, and $0.2 \%(\mathrm{v} / \mathrm{v})$ Triton X-100. Fixed samples were transferred into the staining solution [GUS staining buffer with $20 \%(\mathrm{v} / \mathrm{v})$ methanol, and $1 \mathrm{mM} \mathrm{X}$-Gluc] and slowly vacuumed. After $12 \mathrm{~h}$ incubation at $37^{\circ} \mathrm{C}$ with gentle agitation, samples were rinsed in $70 \%$ ethanol for imaging. At least five clones for every promoter::GUS line were used for GUS staining. All experiments were repeated at least three times with similar results.

\section{Protein localization analysis}

Tobacco leaf samples were analyzed 48 to $72 \mathrm{~h}$ after infiltration. Fluorescence of plant epidermal cells was observed using a LSM 510 confocal laser scanning microscope (Carl Zeiss AG, Oberkochen, Germany). Detection of fluorescence was performed as follows: fluorescence imaging of YFP excitation at $514 \mathrm{~nm}$; scanning at $520-555 \mathrm{~nm}$.

\section{Availability of supporting data}

The phylogenetic trees generated in this study are available in Treebase under study number 15612 (http://treebase. org/treebase-web/search/study/summary.html?id=15612).

\section{Additional files}

Additional file 1: Figure S1. Sequence comparison of poplar WOX family proteins. A. Alignment of the HD sequences. Asterisks indicate residues that are highly conserved in HDs. B. Alignment of the WUS box that is located downstream of HDs. Note that no WUS box was found in PtrWOX13, 9, and 11/12 sub-classes. C. Alignment of the EAR-like domains from WUS and WOX5 proteins. Figure S2. Chromosomal location of PtrWOX genes. The schematic diagram of 18 PtrWOX genes in 14 chromosomes. Homologous blocks derived from the segmental duplication are indicated using the same colors. The diagram of the genome-wide chromosome organization resulting from genome duplication events in $P$. trichocarpa is adapted from Tuskan et al. [31]. Figure S3. Expression analysis of PagWOX genes in different tissues. A. Heat map of hierarchical clustering of PagWOX genes in vegetative tissues ( $\mathrm{YL}$, young leaves; $\mathrm{ML}$, mature leaves; $\mathrm{PS}$, primary stem; SS, secondary stem; R, roots). The data was obtained from our unpublished RNA-seq data. The expression level of genes was determined based on the value of RPKM (reads per kilobase of exon region in a gene per million mapped reads). The clustering was made on expression pattern. Details of the RPKM are shown in Table S3. Color scale represents log2 expression values. Figure S4. The stages of AS and AR regeneration. A-F Leaf explants in indicated stages of AS regeneration. B1-B6 represent the status of explants on 0, 6, 9, 12, 15, 18 days after AS induction. G-J Stem segments in indicated stages of AR regeneration. R1-R4 represent the stem status on $0,3,6,9$ days after AR induction. Bar represents $1 \mathrm{~cm}$ in A-J.

Additional file 2: Table S1. Primer sequences for amplification of PtoWOXs and promoters and qPCR analysis. Table S2. Similarity of WOX sequences from $P$. trichocarpa and $P$. tomentosa. Table S3. RPKM value of PagWOX genes in vegetative tissues obtained from RNA-seq data. Table S4. Accession number of proteins analyzed in this study.

\section{Competing interest}

The authors declare that they have no competing interests.

\section{Authors' contributions}

BL carried out all the constructions, transformation and data collection. LW performed most of the quantitative RT-PCR and data analysis. JZ and JL helped in poplar materials collection and total RNA extraction. $\mathrm{HZ}$ helped in experiment design, data interpretation and manuscript preparation. ML and $J C$ conceived the project, supervised the analysis and critically revised the manuscript. All authors read and approved the final manuscript.

\section{Acknowledgements}

This work was supported by the National High Technology Research and Development Program of China [2013AA102702] to JC, and the National Basic Research Program of China [2012CB1 14500] and a Collaborative Innovation Plan of Jiangsu Higher Education to ML.

\section{Author details}

'Key Laboratory of Forest Genetics \& Biotechnology of Ministry of Education, Nanjing Forestry University, Nanjing 210 037, China. ${ }^{2}$ State Key Laboratory of Tree Genetics and Breeding, Research Institute of Forestry, Chinese Academy of Forestry, Beijing 100 091, China. ${ }^{3}$ Key Laboratory of Non-wood Forest Product of State Forestry Administration, School of Forestry, Central South University of Forestry and Technology, Changsha 410 004, China. ${ }^{4}$ Department of Biology, McGill University, 1205 Dr Penfield Avenue, Montreal, Quebec H3A 1B1, Canada.

Received: 24 December 2013 Accepted: 15 April 2014 Published: 21 April 2014

\section{References}

1. Gehring WJ, Qian YQ, Billeter M, Furukubo-Tokunaga K, Schier AF, Resendez-Perez D, Affolter M, Otting G, Wüthrich K: Homeodomain-DNA recognition. Cell 1994, 78:211-223.

2. Garber R, Kuroiwa A, Gehring WJ: Genomic and CDNA clones of the homeotic locus Antennapedia in Drosophila. EMBO J 1983, 2:2027-2036.

3. Gehring WJ: Exploring the homeobox. Gene 1993, 135:215-221.

4. Ariel FD, Manavella PA, Dezar CA, Chan RL: The true story of the HD-Zip family. Trends Plant Sci 2007, 12:419-426.

5. Vollbrecht E, Veit B, Sinha N, Hake S: The developmental gene Knotted-1 is a member of a maize homeobox gene family. Nature 1991, 350:241-243.

6. Van Der Graaff E, LauX T, Rensing SA: The WUS homeobox-containing (WOX) protein family. Genome Biol 2009, 10:248.

7. Haecker A, Gross-Hardt R, Geiges B, Sarkar A, Breuninger H, Herrmann M, LauX T: Expression dynamics of WOX genes mark cell fate decisions during early embryonic patterning in Arabidopsis thaliana. Development 2004, 131:657-668.

8. Lin H, Niu L, McHale NA, Ohme-Takagi M, Mysore KS, Tadege M: Evolutionarily conserved repressive activity of WOX proteins mediates leaf blade outgrowth and floral organ development in plants. Proc Natl Acad Sci 2013, 110:366-371. 
9. Schoof H, Lenhard M, Haecker A, Mayer KFX, Jürgens G, LauX T: The stem cell population of Arabidopsis shoot meristems is maintained by a regulatory loop between the CLAVATA and WUSCHEL genes. Cell 2000, 100:635-644.

10. Sarkar AK, Luijten M, Miyashima S, Lenhard M, Hashimoto T, Nakajima K, Scheres B, Heidstra R, Laux T: Conserved factors regulate signalling in Arabidopsis thaliana shoot and root stem cell organizers. Nature 2007, 446:811-814.

11. Stahl Y, Wink RH, Ingram GC, Simon R: A signaling module controlling the stem cell niche in Arabidopsis root meristem. Curr Biol 2009, 19:909-914.

12. Hirakawa $Y$, Kondo $Y$, Fukuda H: TDIF peptide signaling regulates vascular stem cell proliferation via the WOX4 homeobox gene in Arabidopsis. Plant Cell 2010, 22:2618-2629.

13. Suer S, Agusti J, Sanchez P, Schwarz M, Greb T: WOX4 imparts auxin responsiveness to cambium cells in Arabidopsis. Plant Cell 2011, 23:3247-3259.

14. Breuninger H, Rikirsch E, Hermann M, Ueda M, Laux T: Differential expression of WOX genes mediates apical-basal axis formation in the Arabidopsis embryo. Dev Cell 2008, 14:867-876.

15. Shimizu R, Ji J, Kelsey E, Ohtsu K, Schnable PS, Scanlon MJ: Tissue specificity and evolution of meristematic WOX3 function. Plant Physiol 2009, 149:841-850

16. Park SO, Zheng Z, Oppenheimer DG, Hauser BA: The PRETTY FEW SEEDS2 gene encodes an Arabidopsis homeodomain protein that regulates ovule development. Development 2005, 132:841-849.

17. Ji J, Shimizu R, Sinha N, Scanlon MJ: Analyses of WOX4 transgenics provide further evidence for the evolution of the WOX gene family during the regulation of diverse stem cell functions. Plant Signal Behav 2010, 5:916-920

18. Palovaara J, Hallberg H, Stasolla C, Hakman I: Comparative expression pattern analysis of WUSCHEL-related homeobox 2 (WOX2) and WOX8/9 in developing seeds and somatic embryos of the gymnosperm Picea abies. New Phytol 2010, 188:122-135.

19. Wu X, Dabi T, Weigel D: Requirement of homeobox gene STIMPY/WOX9 for Arabidopsis meristem growth and maintenance. Curr Biol 2005, 15:436-440.

20. Zhao Y, Hu Y, Dai M, Huang L, Zhou DX: The WUSCHEL-related homeobox gene WOX11 is required to activate shoot-borne crown root development in rice. Plant Cell 2009, 21:736-748.

21. Romera-Branchat M, Ripoll JJ, Yanofsky MF, Pelaz S: The WOX13 homeobox gene promotes replum formation in the Arabidopsis thaliana fruit. Plant $J$ 2013, 73:37-49.

22. Deveaux Y, Toffano-Nioche C, Claisse G, Thareau V, Morin H, Laufs P, Moreau $H$, Kreis M, Lecharny A: Genes of the most conserved WOX clade in plants affect root and flower development in Arabidopsis. BMC Evol Biol 2008, 8:291

23. Etchells JP, Provost CM, Mishra L, Turner SR: WOX4 and WOX14 act downstream of the PXY receptor kinase to regulate plant vascular proliferation independently of any role in vascular organisation. Development 2013, 140:2224-2234.

24. Demura $\mathrm{T}$, Ye ZH: Regulation of plant biomass production. Curr Opin Plant Biol 2010, 13:298-303.

25. Nieminen $\mathrm{K}$, Robischon M, Immanen J, Helariutta Y: Towards optimizing wood development in bioenergy trees. New Phytol 2012, 194:46-53.

26. Slavov GT, DiFazio SP, Martin J, Schackwitz W, Muchero W, Rodgers-Melnick E, Lipphardt MF, Pennacchio CP, Hellsten U, Pennacchio LA, Gunter LE, Ranjan P, Vining K, Pomraning KR, Wilhelm $\sqcup$, Pellegrini M, Mockler TC, Freitag M, Geraldes A, El-Kassaby YA, Mansfield SD, Cronk QCB, Douglas CJ, Strauss SH, Rokhsar D, Tuskan GA: Genome resequencing reveals multiscale geographic structure and extensive linkage disequilibrium in the forest tree Populus trichocarpa. New Phytol 2012, 196:713-725.

27. Zhang X, Zong J, Liu J, Yin J, Zhang D: Genome-wide analysis of WOX gene family in rice, sorghum, maize, arabidopsis and poplar. J Integr Plant Biol 2010, 52:1016-1026.

28. Gambino G, Minuto M, Boccacci P, Perrone I, Vallania R, Gribaudo I: Characterization of expression dynamics of WOX homeodomain transcription factors during somatic embryogenesis in Vitis vinifera. J Exp Bot 2010, 62:1089-1101.

29. Nardmann J, Reisewitz P, Werr W: Discrete shoot and root stem cell-promoting WUS/WOX5 functions are an evolutionary innovation of angiosperms. Mol Bio Evol 2009, 26:1745-1755
30. Hedman H, Zhu T, von Arnold S, Sohlberg Jj: Analysis of the WUSCHEL-RELATED HOMEOBOX gene family in the conifer picea abies reveals extensive conservation as well as dynamic patterns. BMC Plant Biol 2013, 13:89.

31. Tuskan GA, DiFazio S, Jansson S, Bohlmann J, Grigoriev I, Hellsten U, Putnam N, Ralph S, Rombauts S, Salamov A, Schein J, Sterck L, Aerts A, Bhalerao RR, Bhalerao RP, Blaudez D, Boerjan W, Brun A, Brunner A, Busov V, Campbell M, Carlson J, Chalot M, Chapman J, Chen GL, Cooper D, Coutinho PM, Couturier J, Covert S, Cronk Q, et al: The genome of black cottonwood, Populus trichocarpa (Torr. \& Gray). Science 2006, 313:1596-1604.

32. Mayer KFX, Schoof H, Haecker A, Lenhard M, Jürgens G, Laux T: Role of WUSCHEL in regulating stem cell fate in the Arabidopsis shoot meristem. Cell 1998, 95:805-815.

33. Nakata M, Matsumoto N, Tsugeki R, Rikirsch E, Laux T, Okada K: Roles of the middle domain-specific WUSCHEL-RELATED HOMEOBOX genes in early development of leaves in Arabidopsis. Plant Cell 2012, 24:519-535.

34. Wu X, Chory J, Weigel D: Combinations of WOX activities regulate tissue proliferation during Arabidopsis embryonic development. Dev Biol 2007, 309:306-316.

35. Matsumoto N, Okada K: A homeobox gene, PRESSED FLOWER, regulates lateral axis-dependent development of Arabidopsis flowers. Genes Dev 2001, 15:3355-3364.

36. Cheng ZI, Wang L, Sun W, Zhang Y, Zhou C, Su YH, Li W, Sun TT, Zhao XY, Li XG, Cheng Y, Zhao Y, Xie Q, Zhang XS: Pattern of auxin and cytokinin responses for shoot meristem induction results from the regulation of cytokinin biosynthesis by AUXIN RESPONSE FACTOR3. Plant Physiol 2012 161:240-251.

37. Nardmann J, Ji J, Werr W, Scanlon MJ: The maize duplicate genes narrow sheath1 and narrow sheath2 encode a conserved homeobox gene function in a lateral domain of shoot apical meristems. Development 2004, 131:2827-2839.

38. Ji J, Strable J, Shimizu R, Koenig D, Sinha N, Scanlon MJ: WoX4 promotes procambial development. Plant Physiol 2009, 152:1346-1356.

39. Hirakawa Y, Shinohara H, Kondo Y, Inoue A, Nakanomyo I, Ogawa M, Sawa S, Ohashi-Ito K, Matsubayashi Y, Fukuda H: Non-cell-autonomous control of vascular stem cell fate by a CLE peptide/receptor system. Proc Natl Acad Sci 2008, 105:15208-15213.

40. Thompson JD, Gibson TJ, Plewniak F, Jeanmougin F, Higgins DG: The CLUSTAL_X windows interface: flexible strategies for multiple sequence alignment aided by quality analysis tools. Nucleic Acids Res 1997, 25:4876-4882

41. Guindon S, Gascuel O: A simple, fast, and accurate algorithm to estimate large phylogenies by maximum likelihood. Syst Biol 2003, 52:696-704.

42. Jones DT, Taylor WR, Thornton JM: The rapid generation of mutation data matrices from protein sequences. Comput Appl Biosci 1992, 8:275-282.

43. Guo AY, Zhu QH, Chen X, Luo JC: GSDS: a gene structure display server. Yi Chuan 2007, 29:1023-1026.

44. Bailey TL, Williams N, Misleh C, Li WW: MEME: discovering and analyzing DNA and protein sequence motifs. Nucleic Acids Res 2006, 34:369-373.

\section{doi:10.1186/1471-2164-15-296}

Cite this article as: Liu et al:: WUSCHEL-related Homeobox genes in Populus tomentosa: diversified expression patterns and a functional similarity in adventitious root formation. BMC Genomics 2014 15:296.

\section{Submit your next manuscript to BioMed Central and take full advantage of:}

- Convenient online submission

- Thorough peer review

- No space constraints or color figure charges

- Immediate publication on acceptance

- Inclusion in PubMed, CAS, Scopus and Google Scholar

- Research which is freely available for redistribution 